\title{
Comparative Analysis of Fluctuating Asymmetry between Ploidy and Sex in Marine Medaka, Oryzias dancena
}

\author{
${ }^{\dagger}$ In-Seok Park ${ }^{1}$ and Hyun Woo Gil ${ }^{2}$ \\ ${ }^{l}$ Division of Marine Bioscience, College of Ocean Science and Technology, Korea Maritime and Ocean University, \\ Busan 49112, Korea \\ ${ }^{2}$ Bio-Monitoring Center, Sejong 30121, Korea
}

\begin{abstract}
The purpose of this study is to examine fluctuating asymmetry of eye diameter, maxilla length, operculum length, and the number of pectoral fin ray and pelvic fin ray between ploidy and sex in diploid and triploid marine medaka, Oryzias dancena. In all experimental groups, eye diameter and maxilla length showed no significant difference between left side and right side $(p>0.05)$. Results of operculum length in triploid male group and pectoral fin ray's number in diploid male group showed similarity ones with results of operculum length in triploid female group and pectoral fin ray's number in diploid female group $(p<0.05)$. However, the operculum length in diploid male group and pectoral fin ray's number in triploid male group showed consinderable difference with those of operculum length in diploid female group and pectoral fin in triploid female group. Findings of pelvic fin ray's number in all groups were similar to those of pectoral fin ray's number in all groups $(p<0.01)$.
\end{abstract}

Key words : Fluctuating asymmetry, Marine medaka (Oryzias dancena), Ploidy, Sex

\section{INTRODUCTION}

The marine medaka, Oryzias dancena (Beloniformes; Teleostei) is a euryhaline teleost that mainly inhabits the brackish or freshwater of river mouths and estuaries around Bengal Bay and the Malay Peninsula (Roberts, 1998). In hyperosmotic environments, the marine medaka showed better tolerance than the Japanese medaka, O. latipes, including survival rates of adult fish and hatching rates of oosperm (Inoue \& Takei, 2003; Kang et al., 2008). Detailed information on marine medaka biology has begun to be exploited, especially regarding early gonadogenesis, sex differentiation, early ontogenesis, and embryogenesis (Song et al., 2009a, 2009b). In addition, Cho et al. (2010) researched tolerance capacity to salinity changes in this species. This species is highly capable of hyper-osmoregulation as well as hypo-osmoregulation. Recently, the anesthetic effects of clove oil and lidocaine- $\mathrm{HCl}$ on marine medaka were reported by Park et al. (2011). Anesthetic effects on experimental groups of different sizes of marine medaka were observed, and both anesthetic time and recovery time for the juvenile group were shorter than those of the adult group. Kim et al. (2011) reported the characteristics of two functional glucocorticoid receptors in this species.

Triploidization is a technique used to generate sterile aquatic animals by taking advantage of the incompatibility

\footnotetext{
Manuscript received July 18, 2018, Received in revised form August 16, 2018, Accepted September 3, 2018

${ }^{\dagger}$ Corresponding Author : In-Seok Park, Division of Marine Bioscience, College of Ocean Science and Technology, Korea Maritime and Ocean University, Busan 49112, Korea. Tel: +82-51-410-4321, Fax: +82-51-404-4750, E-mail: ispark@kmou.ac.kr
}

This is an Open Access article distributed under the terms of the Creative Commons Attribution Non-Commercial License (http:// creative-commons.org/licenses/by-nc/3.0) which permits unrestricted non-commercial use, distribution, and reproduction in any medium, provided the original work is properly cited. 
in pairing the three homologous chromosomes during meiosis I (Don \& Avtalion, 1986; Park et al., 2016b). This technique has also been used to enhance the productivity of several fish species because of its assumed ability to increase yield by channeling the energy required for gonadal development into somatic growth (Tave, 1993; Park et al., 2016a). More importantly, it generates fish that are unable to breed but contribute to the local gene pool if they were to accidentally escape from confinement. By conferring sterility of exotic fish for a limited purpose, triploidy can serve as an effective method for reducing or eliminating the environmental risks of genetically modified organisms (Dunham \& Devlin, 1999).

Fluctuating asymmetry occurs when the traits of one side of a supposedly bilaterally symmetrical organism differ in a random way from those of the other sides (Wilkins et al., 1995). It is shown as a difference in the number, size, shape or other features of the trait between the left and right side. Developmental homeostasis normally maintains fluctuating asymmetry at a low level. When fluctuating asymmetry increases, it is thought to show a destabilization of development, either by endogenous genetic factors or by exogenous factors such as environmental stress. There are considerably numerous debates over the precise relationship of genetic factors and developmental stability as shown in fluctuating asymmetry, with two major hypotheses (the heterozygosity hypothesis and the genomic coadaptation hypothesis) being distinguished (Wilkins et al., 1995). The former represents that highly heterozygous individuals will have greater developmental stability and therefore lower fluctuating asymmetry, than homozygous individuals because heterozygosity may buffer the organism from perturbations affecting the normal developmental processes and pathways. The latter represents that it is coadapted gene complexes (Wilkins et al., 1995) that ensure developmental stability and when such complexes are disrupted, for example by hybridization or gene flow, stability decreases and fluctuating asymmetry is elevated. In this study, we have examined fluctuating asymmetry of eye diameter, maxilla length, operculum length, and the number of pectoral fin ray and pelvic fin ray between ploidy and sex in marine medaka.

\section{MATERIALS AND METHODS}

The experimental group of diploid marine medaka, Oryzias dancena in this study was reared by methods of Park et al. (2011). On 24 September 2017, one hundred fish were quarantined by the male and female categories and habituated in the $100 \mathrm{~L}$ glass aquariums for 3 days. The sex ratio of males and females was 60 males and 40 females. The culture water was dechlorinated and $30 \%$ of water in the aquarium was exchanged every day. Artemia collected from the cultured aquarium were provided to fish every day. To collect eggs, the fish whose standard length was over $25 \mathrm{~mm}$, was used in this experiment and 35 males and 15 females of marine medaka were placed in each of two aquariums, and 1,000 fertilized eggs were collected immediately with net. The fertilized eggs of diploid experimental group $(n=500)$ were reared in 100 L glass aquarium.

The fertilized eggs of triploid experimental group $(\mathrm{n}=500)$ were left to fertilize for $5 \mathrm{~min}$, and were subjected to cold-shock treatment $\left(4^{\circ} \mathrm{C}\right)$ for $60 \mathrm{~min}$ in order to prevent the onset of the second polar body (Park et al., 2016b, 2018). Afterward, the treatment eggs were reared in $100 \mathrm{~L}$ glass aquarium. After 2 months, flowcytometric analysis was performed to estimate the average cellular DNA content of all individuals from triploid marine medaka. After anesthetizing the fish with 100 ppm clove oil (Park et al., 2011), sample tissues for analysis were separated from all individuals of tail fin. One million of tail fin cells were collected and stained using a high-resolution DNA staining kit (Partec GmbH, Germany) under dark condition at room temperature for 15 min (Park et al., 2018). Stained samples were analyzed on Partec PA-II flowcytometer (Partec $\mathrm{GmbH}$, Germany) to determine the relative DNA content. 
The red blood cells (2.8 pg DNA/ nucleus) of mud loach, Misgurnus mizolepis were used as a standard reference. Individuals which were found triploid were quarantined in a $30 \mathrm{~L}$ glass aquarium for experiment (Park et al., 2018).

On 20 May 2017 (270 days after hatched), this study was performed using samples of 100 marine medakas in total, 50 males and 50 females in diploid and triploid, respectively. Mean standard length of used samples was $3.21 \pm 0.49 \mathrm{~cm}$, and mean body weight of used samples was $584.1 \pm 11.67$ mg. Using the method of Park et al. (2011), the samples were anesthetized with $100 \mathrm{ppm}$ clove oil at $26^{\circ} \mathrm{C}$. For the measurement of fluctuating asymmetry, anesthetized samples were fixed in $10 \%$ neutral formalin. To avoid the sampling fish with guts that were distended by large quantities of food, fish were left to starve for $24 \mathrm{hrs}$ before sampling (Park et al., 2001). Fluctuating asymmetry measurements of fixed samples were taken to the nearest $0.1 \mathrm{~cm}$ using digital vernier calipers (CD-20 CP; Mitytoyo, Kawasaki, Japan). As shown in Fig. 1, eye diameter indicates horizontal distance, and maxilla length (length from middle point of maxilla to each tip of maxilla: $1 \times 2$ in Fig. 1), and operculum length (length from middle point of maxilla to each operculum: $1 \times 3$ in Fig. 1) indicates direct distance.

Digital pictures were taken for fixed samples of each group using a microscope camera (Axiocam MR, Carl zeiss, Germany; $n=100$ ). The Axioskop 4.1 image analysis software (Carl Zeiss, Germany) was used to measure meristic characteristics. Meristic characteristics of pectoral fin ray and pelvic fin ray were measured using the pictures. The differences between the groups were analyzed by student's $t$-test using the SPSS statistics package (SPSS 9.0, SPSS Inc., Chicago, IL, USA). Every experiment was conducted in triplicate.

\section{RESULTS}

Table 1 shows the mean value of meristic and morphometric asymmetry in male marine medaka, Oryzias dancena,

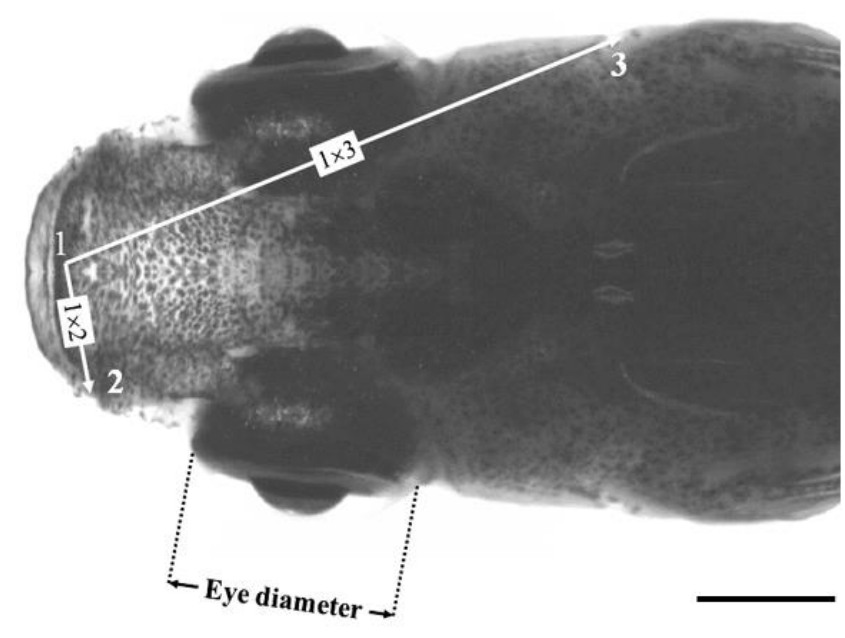

Fig. 1. Morphometric measurements of fluctuating asymmetry in each trait of the marine medaka, Oryzias dancena examined in this study. Eye diameter indicates the horizontal distance. $1 \times 2$ and $1 \times 3$ indicate direct distances. $1 \times 2$, the length from the middle point of the upper lip to each tip of the upper lip; $1 \times 3$, the length from the middle point of the upper lip to each side of the operculum. 1, middle point of the upper lip; 2, tip of the upper lip; 3 , the operculum. Scale bar indicates $2 \mathrm{~mm}$.

and results of $t$-test for difference between diploid and triploid. Eye diameter and maxilla length showed no significant difference between left side and right side in diploid and triploid groups. In diploid and triploid group, operculum length of left side was significantly longer than that of right side $(p<0.01)$. In diploid group, the number of pectoral fin ray's left side outnumbered the pectoral fin ray's right side by ten to nine $(p<0.05)$. Trend of pectoral fin ray's number in triploid group showed opposite disposition with that of pectoral fin ray's number in diploid group $(p<0.01)$. Number of pelvic fin ray in diploid group showed no significant difference between left side and right side. However, the number of pelvic fin ray in triploid group has significant disparity between left side and right side, and the number of pelvic fin ray's right side outnumbered the pelvic fin ray's left side by six to five $(p<0.05)$. 
Table 1. Mean values for the meristic and morphometric asymmetry measurements in male marine medaka, Oryzias dancena, and the results of $t$-tests of the differences between diploid and triploid fish

\begin{tabular}{|c|c|c|c|c|c|c|}
\hline \multirow{2}{*}{ Trait $^{1)}$} & \multicolumn{3}{|c|}{ Diploid } & \multicolumn{3}{|c|}{ Triploid } \\
\hline & Left & Right & $t$-test $\mathrm{t}^{2}$ & Left & Right & $t$-test ${ }^{2)}$ \\
\hline Eye diameter/HL (\%) & 52.2 & 52.2 & NS & 56.4 & 56.4 & NS \\
\hline $1 \times 2 / \operatorname{Ls}(\%)$ & 7.48 & 7.36 & NS & 5.83 & 5.80 & NS \\
\hline $1 \times 3 / \operatorname{Ls}(\%)$ & 27.3 & 26.0 & $* *$ & 23.4 & 22.1 & $* *$ \\
\hline Number of pectoral fin ray & 10 & 9 & $*$ & 9 & 11 & $* *$ \\
\hline Number of pelvic fin ray & 5 & 5 & NS & 5 & 6 & * \\
\hline
\end{tabular}

1) HL, head length; Ls, standard length; $1 \times 2$, length from the middle point of the upper lip to each tip of the upper lip;

$1 \times 3$, length from the middle point of the upper lip to each side of the operculum.

2) NS, denotes not significant at $p=0.05$; *, denotes significant at $p=0.05$; **, denotes significant at $p=0.01$.

Table 2 shows the mean value of meristic and morphometric asymmetry in female marine medaka, and results of $t$-test for difference between diploid and triploid. Eye diameter and maxilla length showed no significant difference between left side and right side in diploid and triploid groups. Operculum length of diploid group showed no significant difference between left side and right side. In triploid group, operculum length of left side was longer than that of right side $(p<0.05)$. In diploid group, the number of pectoral fin ray's left side outnumbered the pectoral fin ray's right side by ten to nine $(p<0.05)$. Results of pec- toral fin ray's number in triploid group were similar to those of pectoral fin ray's number in diploid group ( $p<$ $0.01)$. Also, the number of pectoral fin ray in triploid group had one more than that in diploid group. The number of pelvic fin ray in diploid group showed no significant difference between left side and right side. However, the number of pelvic fin ray in triploid group has significant disparity between left side and right side, and the number of pelvic fin ray's left side outnumbered the pelvic fin ray's right side by six to five $(p<0.05)$.

In diploid and triploid group, eye diameter and maxilla

Table 2. Mean values for the meristic and morphometric asymmetry measurements in female marine medaka, Oryzias dancena, and the results of $\boldsymbol{t}$-tests of the differences between diploid and triploid fish

\begin{tabular}{|c|c|c|c|c|c|c|}
\hline \multirow{2}{*}{ Trait $^{1)}$} & \multicolumn{3}{|c|}{ Diploid } & \multicolumn{3}{|c|}{ Triploid } \\
\hline & Left & Right & $t$-test ${ }^{2)}$ & Left & Right & $t$-test ${ }^{2)}$ \\
\hline Eye diameter/HL (\%) & 53.0 & 53.0 & NS & 62.4 & 62.4 & NS \\
\hline $1 \times 2 / \operatorname{Ls}(\%)$ & 6.80 & 6.76 & NS & 6.03 & 6.03 & NS \\
\hline $1 \times 3 / \operatorname{Ls}(\%)$ & 27.6 & 27.6 & NS & 25.0 & 23.7 & $*$ \\
\hline Number of pectoral fin ray & 10 & 9 & $*$ & 11 & 10 & $* *$ \\
\hline Number of pelvic fin ray & 5 & 5 & NS & 6 & 5 & * \\
\hline
\end{tabular}

${ }^{1)} \mathrm{HL}$, head length; Ls, standard length; $1 \times 2$, length from the middle point of the upper lip to each tip of the upper lip;

$1 \times 3$, length from the middle point of the upper lip to each side of the operculum.

${ }^{2)} \mathrm{NS}$, denotes not significant at $p=0.05$; *, denotes significant at $p=0.05 ; * *$, denotes significant at $p=0.01$. 
length's trends of female marine medaka were similar to those of male marine medaka (Tables 1 and 2). Operculum length of diploid male group showed significant difference between left side and right side, but that of diploid female group showed no significant difference between left side and right side. Results of operculum length in triploid male group displayed similar propensity with those of operculum length in triploid female group. Findings of pectoral fin ray's number in diploid male group showed similar propensity with the results of pectoral fin ray's number in diploid female group, however, the pectoral fin ray's number in triploid male group showed consinderable difference with that of pectoral fin ray in triploid female group. Also, the results of pelvic fin ray's number in all groups showed similarity with those of pectoral fin ray's number in all groups.

\section{DISCUSSION}

According to Soule (1967), if heterozygosity or genomic coadaptation mediates developmental stability, then populations which are highly asymmetrical in one character are likely to be asymmetrical in other characters. On this basis, asymmetries in different traits are often combined to make an overall population asymmetry parameter (PAP; Soule, 1967). The meristic and morphometric traits analyzed here appear to represent two distinct characteristic sets. The meristic traits showed elevated fluctuating asymmetry in hybrids but these decreased when hybrids became triploid. The morphometric traits did not show elevated fluctuating asymmetry in hybrids and were relatively unaffected by triploidisation. There was no consistent, significant correlation to fluctuating asymmetry in those two characteristic sets among individual salmon or individual hybrids.

In the previous paper, Claytor and Verspoor (1991) has found that variation in meristic and morphometric characteristics of sympatric Atlantic salmon, Salmo salar populations was discordant and variations in them were likely to be congruent only by coincidence. It seems reasonable to conclude that the expression of these characteristic sets in salmon is under separate genetic control. This is consistentistic with the observation that meristic traits are determined during embryonic development before hatching (Taning, 1950; Barlow, 1961) whereas morphometric traits remain unstable during later growth, also their phenotype is strongly influenced by rearing conditions, especially stream gradient (Claytor et al., 1991). Therefore, it seems unacceptable, at least as far as Atlantic salmon is concerned, to combine those two kinds of traits in analysis of fluctuating asymmetry as is done with other species (see, for example, Soule 1967; Graham \& Felley, 1985).

In our study, triploid marine medaka, Oryzias dancena was significantly different from that of diploid in some factors of fluctuating asymmetry. According to Wilkins et al. (1995), triploid salmon was not significantly different from their diploid siblings in fluctuating asymmetry, although their overall values were lower. Consequently, the presence of the extra chromosomal set had no significant effect on developmental stability. In triploid hybrids, in contrast, duplication of the maternal set of salmon chromosomes restores the relational balance absent in diploid hybrids, resulting in a statistically significant reduction in fluctuating asymmetry and accordingly greater developmental stability. In their study of rainbow trout, Oncorhynchus mykiss, Leary et al. (1985) also observed a significant reduction in fluctuating asymmetry due to triploidisation (Wilkins et al., 1995). Future investigations of asymmetry in marine medaka are sure to focus on the directional asymmetry of gonad development between male and female diploid marine medaka.

\section{ACKNOWLEDGEMENTS}

The authors would like to thank anonymous editors for their helpful suggestions that improved the quality of this paper. We declare that all the experiments performed in 
this study complied with the current laws of Korea (Ordinance of Agriculture, Food and Fisheries, No. 1-the law regarding experimental animals, No. 9982) and the Ethical Guidelines of Korea Maritime and Ocen University, Korea.

\section{REFERENCES}

Barlow GW (1961) Causes and significance of morphological variation in fishes. Syst Biol 10:105-117.

Cho YS, Lee SY, Kim DS, Nam YK (2010) Tolerance capacity to salinity changes in adult and larva of Oryzias dancena, a euryhaline medaka. Korean J Ichthyol 22:9-16.

Claytor RR, MacCrimmon HR, Gots BL (1991) Continental and ecological variance components of European and North American Atlantic salmon (Salmo salar) phenotypes. Biol J Linn Sot 44:203-229.

Claytor RR, Verspoor E (1991) Discordant phenotypic variation in sympatric resident and anadromous Atlantic salmon (Salmo salar) populations. Can J Zool 69: 2846-2852.

Don J, Avtalion RR (1986) The induction of triploidy in Oreochromis aureus by heat shock. Theor Appl Genet 72:186-192.

Dunham RA, Devlin RH (1999) Comparison of traditional breeding and transgenesis in farmed fish with implications for growth enhancement and fitness. In: Murray JD, Anderson GB, Oberbauer AM, McGloughlin MN (eds), Transgenic Animals in Agriculture, CAB International, New York, pp 209-229.

Graham JH, Felley JD (1985) Genomic coadaptation and developmental stability within introgressed populations of Enneacunthus gloriosus and E. obesus (Pisces, Centrarchidae). Evolution 39:104-114.

Inoue K, Takei Y (2003) Asian medaka fishes offer new models for studying mechanisms of seawater adaptation. Comp Biochem Physiol B 136:635-645.

Kang CK, Tsai SC, Lee TH, Hwang PP (2008) Differential expression of branchial $\mathrm{Na}^{+} / \mathrm{K}^{+}$-ATPase of two medaka species (Oryzias latipes) and (Oryzias dancena), with different salinity tolerances acclimated to fresh water, brackish water and seawater. Comp Biochem Phys A $151: 566-575$.

Kim MA, Kim DS, Sohn YC (2011) Characterization of two functional glucocorticoid receptors in the marine medaka, Oryzias dancena. Gen Comp Endocrinol 171: 341-349.

Leary RF, Allendorf FW, Knudsen KL, Thorgaard GH (1985) Heterozygosity and developmental stability in gynogenetic diploid and triploid rainbow trout. Heredity $54: 219-225$.

Park I-S, Gil HW, Kim DS (2018) Morphometric characteristics of diploid and triploid marine medaka, Oryzias dancena. Dev Reprod 22:183-192.

Park I-S, Gil HW, Lee TH, Nam YK, Kim DS (2016a) Comparative study of growth and gonad maturation in diploid and triploid marine medaka, Oryzias dancena. Dev Reprod 20:305-314.

Park I-S, Gil HW, Lee TH, Nam YK, Ko MG, Kim DS (2016b) Cytogenetic study of diploid and triploid marine medaka, Oryzias dancena. Korean J Ichthyol 28: 215-222.

Park I-S, Im JH, Ryu DK, Nam YK, Kim DS (2001) Effect of starvation on morphometric changes in Rhynchocypris oxycephalus (Sauvage and Dabry). J Appl Ichthyol 17:277-281.

Park I-S, Park SJ, Gil HW, Nam YK, Kim DS (2011) Anesthetic effects of clove oil and lidocaine- $\mathrm{HCl}$ on marine medaka, Oryzias dancena. Lab Animal 40:45-51.

Roberts TR (1998) Systematic observations on tropical Asian medakas or ricefishes of genus Oryzias, with descriptions of four new species. Ichthyol Res 45:213-224.

Song HY, Nam YK, Bang I-C, Kim DS (2009a) Early gonadogenesis and sex differentiation of marine medaka, Oryzias dancena (Beloniformes; Teleostei). Korean J Ichthyol 21:141-148 (in Korean with an English abstract). 
Song HY, Nam YK, Bang I-C, Kim DS (2009b) Embryogenesis and early ontogenesis of a marine medaka, Oryzias dancena. Korean J Ichthyol 21:227-238 (in Korean with an English abstract).

Soule M (1967) Phenetics of natural populations. II. Asymmetry and evolution in a lizard. Am Natur 101:141-160. Taning AV (1950) Experimental study of meristic charac- ters in fishes. Biol Rev (Cambridge) 271:169-193.

Tave D (1993) Growth of triploid and diploid bighead carp, Hypophthalmichthys nobilis. J Appl Aquacult 2:13-26.

Wilkins NP, Gosling E, Curatolo A, Linnane A, Jordan C, Courtney HP (1995) Fluctuating asymmetry in Atlantic salmon, European trout and their hybrids, including triploids. Aquaculture 137:77-85. 